\title{
Resistance to Artesunate + Mefloquine does not incurin fitness cost in malaria parasites
}

\author{
Louise A Rodrigues, Gisela Henriques*, Pedro Cravo \\ From Parasite to Prevention: Advances in the understanding of malaria \\ Edinburgh, UK. 20-22 October 2010
}

\section{Background}

Resistance to almost all available anti-malarials poses a large threat for the effective control of Malaria. The only remaining exceptions are the artemisinin (ART) derivatives. However, there is now evidence that appearance of resistance to ART either administered alone or in combination with other anti-malarials may become a reality.

Although resistance to treatment is an obstacle for controlling the disease, in many biological models increased drug resistance is often associated with a biological cost. Similarly, in malaria parasites, resistance to anti-malarial drugs such as chloroquine (CQ) [1] and mefloquine (MF) [2] has been reported as causing a reduction in parasite's fitness associated with lower asexual growth rates and/or reduced transmission efficiency.

By using an artesunate (ATN)-resistant parasite, AS-ATN, we have selected a parasite clone (ASATNMF-1) which is resistance to the artesunate + mefloquine (ATN + MF) version of Artemisinin Combination Therapy [3]. AS-ATNMF-1 is also resistant to each component of the combination when administered separately. AS-ATNMF-1 was obtained after twentyseven consecutive sub-inoculations of AS-ATN in mice treated with the ATN + MF combination. Since consecutive sub-inoculations into mice have been shown to cause increased virulence in malaria parasites [4], ASATN was also sub-inoculated twenty-seven times into untreated mice in parallel. The resulting line was named AS-ATN27P.

In order to assess the fitness cost associated with resistance to the combination ATN + MF, AS-ATNMF1 parasites were grown with the AS-ATN parental clone or with AS-ATN27P within the same host in absence of drug pressure, for 14 days.

\footnotetext{
Molecular Biology Unity, Institute of Hygene and Tropical Medicine, Universidade Nova de Lisboa, Portugal
}

The proportions of each parasite parasite within were determined along time by Proportional Sequencing [5].

\section{Results}

When in competition within the same host, AS-ATNMF-1 outgrows the unpassaged progenitor AS-ATN. However the same is not observed when grown in competition with the passaged parasites AS-ATN27P.

\section{Conclusions}

Our data suggest that consecutive passaging may have induced an improvement in AS-ATNMF-1 fitness and that resistance to the combination ATN + MF seem to have no negative impact on the growth of these parasites. These results may have implications regarding the maintenance of ACT-resistant parasites in the absence of drug pressure.

\section{Published: 20 October 2010}

\section{References}

1. Rosario VE, Hall R, Walliker D, Beale GH: Persistence of drug-resistant malaria parasites. Lancet 1978, 1(8057):185-7, Jan 28.

2. Preechapornkul P, Imwong M, Chotivanich K, Pongtavornpinyo W, Dondorp AM, Day NP, White NJ, Pukrittayakamee S: Plasmodium falciparum pfmdr1 amplification, mefloquine resistance, and parasite fitness. Antimicrob Agents Chemother 2009, 53(4):1509-1, Apr.

3. Rodrigues LA, Henriques $G$, Borges ST, Hunt P, Sanchez CP, Martinelli A, Cravo P: Experimental evolution of resistance to Artemisinin Combination Therapy results in amplification of the $m d r 1$ gene in a rodent malaria parasite. Plos One 2010.

4. Mackinnon MJ, Read AF: Selection for high and low virulence in the malaria parasite Plasmodium chabaudi. Proc Biol Sci 1999, 266(1420):741-8, Apr 7.

5. Hunt P, Fawcett R, Carter R, Walliker D: Estimating SNP proportions in populations of malaria parasites by sequencing: valida-tion and applications. Mol Biochem Parasitol 2005, 143(2):173-8, Oct.

doi:10.1186/1475-2875-9-S2-P43

Cite this article as: Rodrigues et al.: Resistance to Artesunate + Mefloquine does not incurin fitness cost in malaria parasites. Malaria Journal 2010 9(Suppl 2):P43 